\title{
Exceptionally Small Attenuation Factors in Molecular Wires
}

Francesco Giacalone, ${ }^{a}$ José L. Segura, ${ }^{a}$ Nazario Martín ${ }^{\star a}$ and Dirk M. Guldi ${ }^{\star b}$ ${ }^{a}$ Departamento de Química Orgánica, Facultad de Química, Universidad Complutense, E-28040 Madrid, Spain.

${ }^{b}$ Radiation Laboratory, University of Notre Dame, IN 46556, USA

\section{Supporting Information}

\section{Spectroscopic Characterization of triad 1-5:}

\section{Experimental Section}

All solvents were dried and distilled according to standard procedures. Reagents were used as purchased. All air-sensitive reactions were carried out under an argon

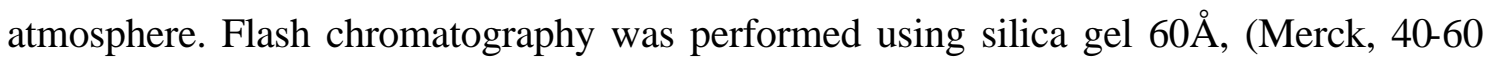
$\mu \mathrm{m})$. Thin layer chromatography (TLC) was performed on silica gel $60 \mathrm{~F}_{254}$ coated aluminum sheets (Merck) with detection by UV at $254 \mathrm{~nm}$. NMR spectra were recorded on Bruker AC-200 ( $\left.{ }^{1} \mathrm{H}: 200 \mathrm{MHz} ;{ }^{13} \mathrm{C}: 50 \mathrm{MHz}\right)$, Bruker AC-300 or Varian XL-300 $\left({ }^{1} \mathrm{H}: 300 \mathrm{MHz} ;{ }^{13} \mathrm{C}: 75 \mathrm{MHz}\right)$ and Bruker DRX-500 or AMX-500 $\left({ }^{1} \mathrm{H}: 500 \mathrm{MHz} ;{ }^{13} \mathrm{C}\right.$ : $125 \mathrm{MHz}$ ) spectrometers at $298 \mathrm{~K}$ using partially deuterated solvents as internal standards. Coupling constants $(J)$ are denoted in $\mathrm{Hz}$ and chemical shifts ( ${ }^{\mathrm{T}}$ ) in ppm. Multiplicities are denoted as follows: $\mathrm{s}=$ singlet, $\mathrm{d}=$ doublet, $\mathrm{t}=$ triplet, $\mathrm{q}=$ quartet, $\mathrm{dd}$ $=$ double doublet, $\mathrm{m}=$ multiplet. FT-IR spectra were recorded on a Nicolet-Magna-IR 5550 spectrometer. Mass spectra by electrospray ionization (ESI) were recorded on a HP1100MSD spectrometer. 
Triad 1: Eluent: toluene / ethyl acetate 9:1; Yield: 17\%; ${ }^{1} \mathrm{H} \mathrm{NMR}\left(\mathrm{CDCl}_{3}, 500 \mathrm{MHz}\right)$ : $\delta=7.83(\mathrm{~s}, 2 \mathrm{H}), 7.70(\mathrm{~m}, 3 \mathrm{H}), 7.59(\mathrm{~d}, 2 \mathrm{H}, J=5 \mathrm{~Hz}), 7.38(\mathrm{~d}, 1 \mathrm{H}, J=5 \mathrm{~Hz}), 7.28(\mathrm{~m}$, 2H), $7.17(\mathrm{~s}, 1 \mathrm{H}), 7.16(\mathrm{~d}, 1 \mathrm{H}, J=16.3 \mathrm{~Hz}), 7.14(\mathrm{~d}, 1 \mathrm{H}, J=16.3 \mathrm{~Hz}), 6.29(\mathrm{~s}, 4 \mathrm{H}), 5.35$ (s, 1H, $\left.\mathrm{CH}_{2}-\mathrm{N}-\right), 5.30(\mathrm{~s}, 1 \mathrm{H}) ; 3.99(\mathrm{~s}, 1 \mathrm{H}), 3.82$ (d, 2H, J= $\left.4 \mathrm{~Hz}\right), 3.79$ (d, 2H, J=4 Hz), $3.72(\mathrm{t}, 4 \mathrm{H}, J=5 \mathrm{~Hz}), 3.57(\mathrm{t}, 4 \mathrm{H}, J=5 \mathrm{~Hz}), 3.36(\mathrm{~s}, 3 \mathrm{H}) .{ }^{13} \mathrm{C} \mathrm{NMR}\left(\mathrm{CDCl}_{3}, 125 \mathrm{MHz}\right)$. $\delta=147.29,146.26,146.13,145.89,145.53,145.29,144.70,144.56,144.31,143.09$, $142.95,142.55,142.14,142.04,141.60,141.46,140.09,139.87,139.55,137.84$ $135.80,135.65,135.52,135.22,134.88,134.77,130.00,129.71,129.19,128.99$, $128.19,128.00,126.95,125.98,125.27,124.91,124.38,122.86,121.99,117.23$, $117.08,82.30,71.99,70.68,70.64,70.60,68.82,65.75,59.08,52.39,48.67$. FTIR $(\mathrm{KBr}): \mathrm{v}=3434,2921,2851,1632,1508,1455,1107,527 \mathrm{~cm}^{-1} . \mathrm{MS} . \mathrm{m} / \mathrm{z}(\mathrm{ESI})=1389$ $\left(\mathrm{M}^{+}, 100\right), 1219$ (65), 551 (62). UV-Vis $\left(\mathrm{CH}_{2} \mathrm{Cl}_{2}\right) \lambda_{\text {máx }}(\log \varepsilon): 257$ (6.85), 326 (6.58), 378 (6.29), 432 (shoulder, 6.14), 441 (6.13). M.p.: >300 ${ }^{\circ} \mathrm{C}$

Triad 2: Eluent: hexane 1/ toluene 1. Yield: $30 \% .{ }^{1} \mathrm{H}$ NMR $\left(\mathrm{CDCl}_{3} / \mathrm{CS}_{2}\right) . \delta=8.33-$ $8.22(\mathrm{~m}, 4 \mathrm{H}) ; 7.87-7.63(\mathrm{~m}, 6 \mathrm{H}) ; 7.53(\mathrm{~d}, 2 \mathrm{H}) ; 7.43\left(\mathrm{~d}, 1 \mathrm{H}, J^{3}=16.4 \mathrm{~Hz}\right) ; 7.22(\mathrm{~d}, 1 \mathrm{H}$, $\left.J^{3}=16.4 \mathrm{~Hz}\right) ; 7.08\left(\mathrm{~d}, 2 \mathrm{H}, J^{3}=16.6 \mathrm{~Hz}\right) ; 7.04(\mathrm{~s}, 1 \mathrm{H}) ; 6.76(\mathrm{~s}, 4 \mathrm{H}) ; 5.10(\mathrm{~d}, 1 \mathrm{H}, J=$ $9.2 \mathrm{~Hz}) ; 5.05(\mathrm{~s}, 1 \mathrm{H}) ; 4.12(\mathrm{~d}, 1 \mathrm{H} J=9.2 \mathrm{~Hz}) ; 4.04(\mathrm{~m}, 4 \mathrm{H}) ; 3.25(\mathrm{~m}, 1 \mathrm{H}) ; 2.56(\mathrm{~m}, 1 \mathrm{H})$; $1.88(\mathrm{~m}, 6 \mathrm{H}) ; 1.55-1.24(\mathrm{~m}, 22 \mathrm{H}) ; 0.92(\mathrm{~m}, 9 \mathrm{H}) .{ }^{13} \mathrm{C} \mathrm{NMR}\left(\mathrm{CDCl}_{3} / \mathrm{CS}_{2}\right) . \delta=156.26$, $153.97,153.30,151.30,150.74,147.08,146.60,146.26,146.03,145.72,145.57$, $145.30,145.03,144.48,144.18,143.78,142.96,142.80,142.38,142.09,141.93$, $141.81,141.49,141.35,140.00,139.74,139.37,137.74,136.67,136.51,136.39$, $135.64,135.53,133.80,133.67,133.57,133.39,131.61,130.83,129.56,128.69$, $127.74,126.99,126.62,125.43,124.86,123.48,118.21,110.53,109.95,82.28,69.06$, 
$68.69,66.72,32.04,31.70,29.81,29.49,28.50,27.66,26.02,22.91,22.84,14.28$, 14.16. MS. $\mathrm{m} / \mathrm{z}(\mathrm{ESI})=1659\left(\mathrm{MH}^{+}, 100\right), 1573(52), 1432(17)$. FTIR $(\mathrm{KBr}): \mathrm{v}=2921$, 2853, 1632, 1505, 1460, 1107, $527 \mathrm{~cm}^{-1}$. UV-Vis $\left(\mathrm{CH}_{2} \mathrm{Cl}_{2}\right) \lambda_{\max }(\mathrm{nm}): 229,256,330$, 403 (shoulder), 431, 455. M.p.: >300 ${ }^{\circ} \mathrm{C}$.

Triad 3: Eluent: cyclohexane / toluene 3:7; Yield: 19\%. ${ }^{1} \mathrm{H} \mathrm{NMR}\left(\mathrm{CDCl}_{3} / \mathrm{CS}_{2}, 500\right.$ MHz): $\delta=8.29(\mathrm{~m}, 2 \mathrm{H}), 7.89(\mathrm{~m}, 2 \mathrm{H}), 7.80(\mathrm{t}, 3 \mathrm{H}, J=5 \mathrm{~Hz}), 7.62(\mathrm{~m}, 1 \mathrm{H}), 7.53(\mathrm{~m}$, $3 \mathrm{H}), 7.47(\mathrm{~m}, 2 \mathrm{H}), 7.44(\mathrm{t}, 2 \mathrm{H}, J=5 \mathrm{~Hz}), 7.41(\mathrm{~s}, 1 \mathrm{H}), 7.35(\mathrm{~s}, 1 \mathrm{H}), 7.23(\mathrm{~m}, 2 \mathrm{H}), 7.05$ $\left(\mathrm{d}, 2 \mathrm{H}, J_{\text {trans }}=16.7 \mathrm{~Hz}\right), 7.03(\mathrm{~s}, 1 \mathrm{H}), 7.01\left(\mathrm{~d}, 1 \mathrm{H}, J_{\text {trans }}=16.7 \mathrm{~Hz}\right), 6.31(\mathrm{~s}, 4 \mathrm{H}), 5.01(\mathrm{~d}$, $\left.1 \mathrm{H}, J=9.5 \mathrm{~Hz}, \mathrm{CH}_{2}-\mathrm{N}-\right), 4.97$ (s, $\left.1 \mathrm{H},-\mathrm{CH}-\mathrm{N}-\right), 4.30$ (d, $\left.1 \mathrm{H}, J=9.5 \mathrm{~Hz}, \mathrm{CH}_{2}-\mathrm{N}-\right), 4.04$ (t, $4 \mathrm{H}, J=6 \mathrm{~Hz}), 2.87\left(\mathrm{~s}, 3 \mathrm{H}, \mathrm{N}-\mathrm{CH}_{3}\right), 1.89(\mathrm{~m}, 4 \mathrm{H}), 1.58(\mathrm{~m}, 4 \mathrm{H}), 1.57-1.27(\mathrm{~m}, 8 \mathrm{H}), 0.97$ $(\mathrm{m}, 6 \mathrm{H}) .{ }^{13} \mathrm{C} \mathrm{NMR}\left(\mathrm{CDCl}_{3} / \mathrm{CS}_{2}, 125 \mathrm{MHz}\right) . \delta=156.04,153.85,153.34,153.10,151.06$, $147.31,146.73,146.36,146.25,146.18,145.97,145.66,145.59,145.41,145.28$, $144.74,144.70,144.42,143.20,143.05,142.73,142.63,142.28,142.20,142.09$, $141.97,141.73,141.61,140.27,140.03,139.68,138.63,138.37,136.98,136.70$, $135.87,135.79,135.43,133.93,133.80,133.73,132.74,132.07,131.57,131.35$, $129.63,129.37,128.05,127.60,127.26,127.08,127.02,126.76,126.63,126.54$, $126.39,126.02,124.85,124.26,124.18,124.13,118.43,118.27,110.23,83.10,76.85$, 69.66, 68.89, 68.58, 39.75, 32.10, 29.93, 26.42, 23.28, 14.57. FTIR $(\mathrm{KBr}): v=2923$, 2852, 1633, 1556, 1421, 1033, 876, 796, 526, $468 \mathrm{~cm}^{-1} . \mathrm{MS} . \mathrm{m} / \mathrm{z}(\mathrm{ESI})=1661\left(\mathrm{M}^{+}, 95\right)$, 1619 (100), 1577 (62), 1332 (37), 806 (31). UV-Vis $\left(\mathrm{CH}_{2} \mathrm{Cl}_{2}\right) \lambda_{\text {máx }}(\log \varepsilon)$ : 256 (5.03), 327 (4.67), 413 (4.59). M.p.: $>300{ }^{\circ} \mathrm{C}$.

Triad 4: Eluent: toluene / ethyl acetate 9:1. Yield: 27\%. ${ }^{1} \mathrm{H}$ NMR $\left(\mathrm{CDCl}_{3} / \mathrm{CS}_{2}, 500\right.$ MHz): $\delta=8.42(\mathrm{~m}, 1 \mathrm{H}), 8.26(\mathrm{~m}, 2 \mathrm{H}), 7.91(\mathrm{~m}, 2 \mathrm{H}), 7.80(\mathrm{~m}, 1 \mathrm{H}), 7.66(\mathrm{t}, 2 \mathrm{H}, J=7$ 
$\mathrm{Hz}), 7.55(\mathrm{~d}, 4 \mathrm{H}, J=7 \mathrm{~Hz}), 7.49(\mathrm{~m}, 9 \mathrm{H}), 7.38(\mathrm{~m}, 3 \mathrm{H}), 7.27(\mathrm{~m}, 4 \mathrm{H}), 7.10(\mathrm{~m}, 7 \mathrm{H})$, $6.32(\mathrm{~s}, 4 \mathrm{H}), 5.35$ (s, 1H, $\left.\mathrm{CH}_{2}-\mathrm{N}-\right), 5.30$ (s, 1H, CH-N-), 4.41 (s, 1H, CH-N-), 4.04 (t, $4 \mathrm{H}, J=6 \mathrm{~Hz}), 3.83(\mathrm{~d}, 2 \mathrm{H}, J=5 \mathrm{~Hz}), 3.79(\mathrm{~d}, 2 \mathrm{H}, J=5 \mathrm{~Hz}), 3.73(\mathrm{t}, 4 \mathrm{H}, J=5 \mathrm{~Hz}), 3.57$ (t, 4H, J= $5 \mathrm{~Hz}), 3.37\left(\mathrm{~s}, 3 \mathrm{H}, \mathrm{N}-\mathrm{CH}_{3}\right), 1.89(\mathrm{~m}, 4 \mathrm{H}), 1.57(\mathrm{~m}, 4 \mathrm{H}), 1.41-1.26(\mathrm{~m}, 8 \mathrm{H})$, $0.89(\mathrm{~m}, 6 \mathrm{H}) .{ }^{13} \mathrm{C} \mathrm{NMR}\left(\mathrm{CDCl}_{3} / \mathrm{CS}_{2}, 125 \mathrm{MHz}\right) . \delta=150.16,146.37,145.56,145.37$, $145.21,144.99,144.88,144.63,144.37,144.31,143.79,143.67,143.41,142.21$, $142.05,141.67,141.25,141.14,141.00,140.70,140.57,139.18,138.98,138.65$, $137.12,137.02,136.65,136.47,136.13,135.52,135.28,135.12,134.75,134.62$, $133.42,132.94,130.87,130.71,130.47,129.83,129.64,129.83,129.10,128.76$, $128.50,128.24,128.06,127.84,127.30,127.08,127.03,126.86,126.58,126.39$, $126.29,125.97,125.56,125.05,124.01,123.47,123.17,122.60,117.24,116.76$, $116.36,109.43,82.28,71.98,70.62,69.34,58.94,52.45,30.87,28.93,28.70,25.19$, 21.94, 13.27. FTIR (KBr): $v=3434,2921,2852,1631,1461,1419,1178,1106,957$, $525 \mathrm{~cm}^{-1}$. MS. m/z (ESI) $=1998\left(\mathrm{M}^{+}, 36\right), 1913(100), 1827(85), 1738$ (35), 1597 (27). UV-Vis $\left(\mathrm{CH}_{2} \mathrm{Cl}_{2}\right) \lambda_{\text {máx }}(\log \varepsilon): 255$ (5.29), 311 (shoulder, 4.96), 333 (4.98), 435 (5.19). M.p.: $216-219^{\circ} \mathrm{C}$ (methanol).

Triad 5: Eluent: hexane / toluene 1:1. Yield: 30\%. ${ }^{1} \mathrm{H}$ NMR $\left(\mathrm{CDCl}_{3} / \mathrm{CS}_{2}\right): \delta=7.79$ $(\mathrm{m}, 3 \mathrm{H}) ; 7.65(\mathrm{~m}, 3 \mathrm{H}) ; 7.56-7.42(\mathrm{~m}, 17 \mathrm{H}), 7.41\left(\mathrm{~d}, 4 \mathrm{H}, J_{\text {trans }}^{3}=16.3 \mathrm{~Hz}\right) ; 7.30(\mathrm{~m}, 2 \mathrm{H})$; $7.13(\mathrm{~s}, 2 \mathrm{H}) ; 7.10(\mathrm{~s}, 4 \mathrm{H}) ; 7.09\left(\mathrm{~d}, 4 \mathrm{H}, J_{\text {trans }}^{3}=15.3 \mathrm{~Hz}\right) ; 7.08\left(\mathrm{~d}, 4 \mathrm{H} ; J^{3}\right.$ trans $\left.=16.3 \mathrm{~Hz}\right)$; $6.32(\mathrm{~s}, 4 \mathrm{H}) ; 5.13(\mathrm{~d}, 1 \mathrm{H}, J=9.2 \mathrm{~Hz}) ; 5.07(\mathrm{~s}, 1 \mathrm{H}) ; 4.14(\mathrm{~d}, 1 \mathrm{H}, J=9.2 \mathrm{~Hz}) ; 4.05(\mathrm{q}$, $12 \mathrm{H}) ; 3.28(\mathrm{~m}, 1 \mathrm{H}) ; 2.59(\mathrm{~m}, 1 \mathrm{H}) ; 1.90(\mathrm{~m}, 14 \mathrm{H}) ; 1.60-1.26(\mathrm{~m}, 46 \mathrm{H}) ; 0.98(\mathrm{~m}, 21 \mathrm{H})$. ${ }^{13} \mathrm{C} \mathrm{NMR}\left(\mathrm{CDCl}_{3} / \mathrm{CS}_{2}\right) . \delta=156.31,154.01,153.36,153.28,150.87,147.09,146.63$, $146.29,145.96,145.74,145.59,145.05,144.50,144.21,142.97,142.82,142.39$, $142.11,141.94,141.83,141.49,141.36,140.00,139.77,139.40,137.95,136.98$, 
$136.65,136.42,136.34,136.25,135.92,135.63,135.08,134.53,129.55,128.10$, $126.70,126.45,126.33,125.88,125.17,124.79,124.15,123.70,123.03,122.74$, $121.95,117.16,117.07,110.15,110.00,82.32,69.16,68.7,66.73,53.39,53.13,32.04$, $31.74,30.13,29.81,29.57,28.50,27.66,26.75,26.06,22.85,14.28,14.16 . \mathrm{MS} . \mathrm{m} / \mathrm{z}$ $(\mathrm{ESI})=2569\left(\mathrm{MH}^{+}, 100\right)$, FTIR $(\mathrm{KBr}): \mathrm{v}=2923,2853,1632,1461,1422,1178,1107$, 957, $527 \mathrm{~cm}^{-1}$. UV-Vis $\left(\mathrm{CH}_{2} \mathrm{Cl}_{2}\right) \lambda_{\max }(\mathrm{nm})(\log \varepsilon): 254$ (5.15), 330 (4.48), 458 (5.19). M.p.: $>300{ }^{\circ} \mathrm{C}$. 


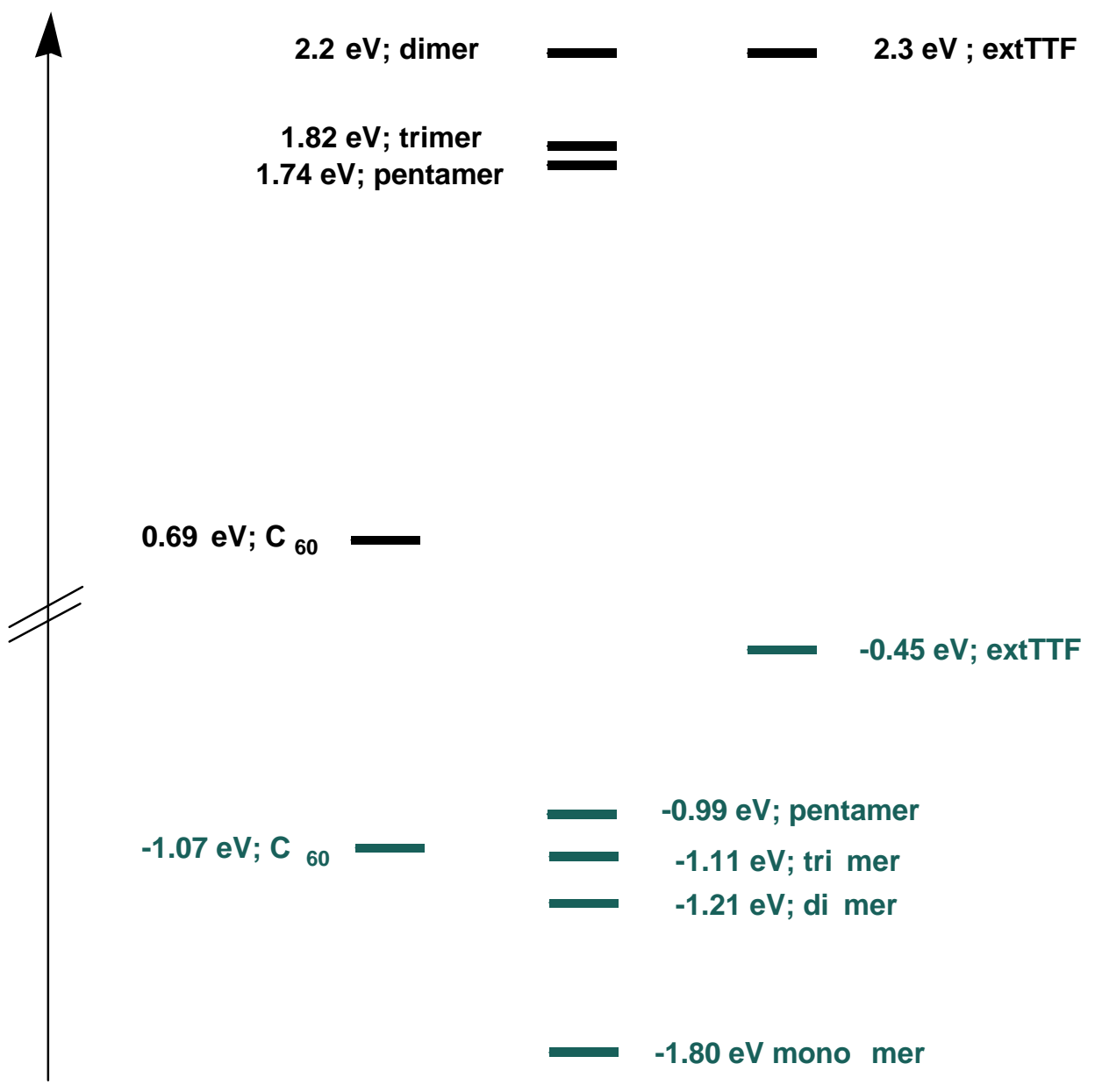

Figure S1. HOMO (dark green) and LUMO (black) levels of $\mathrm{C}_{60}$, oligo-PPVs, and exTTF - determination was carried out analogously to ref. 5. 

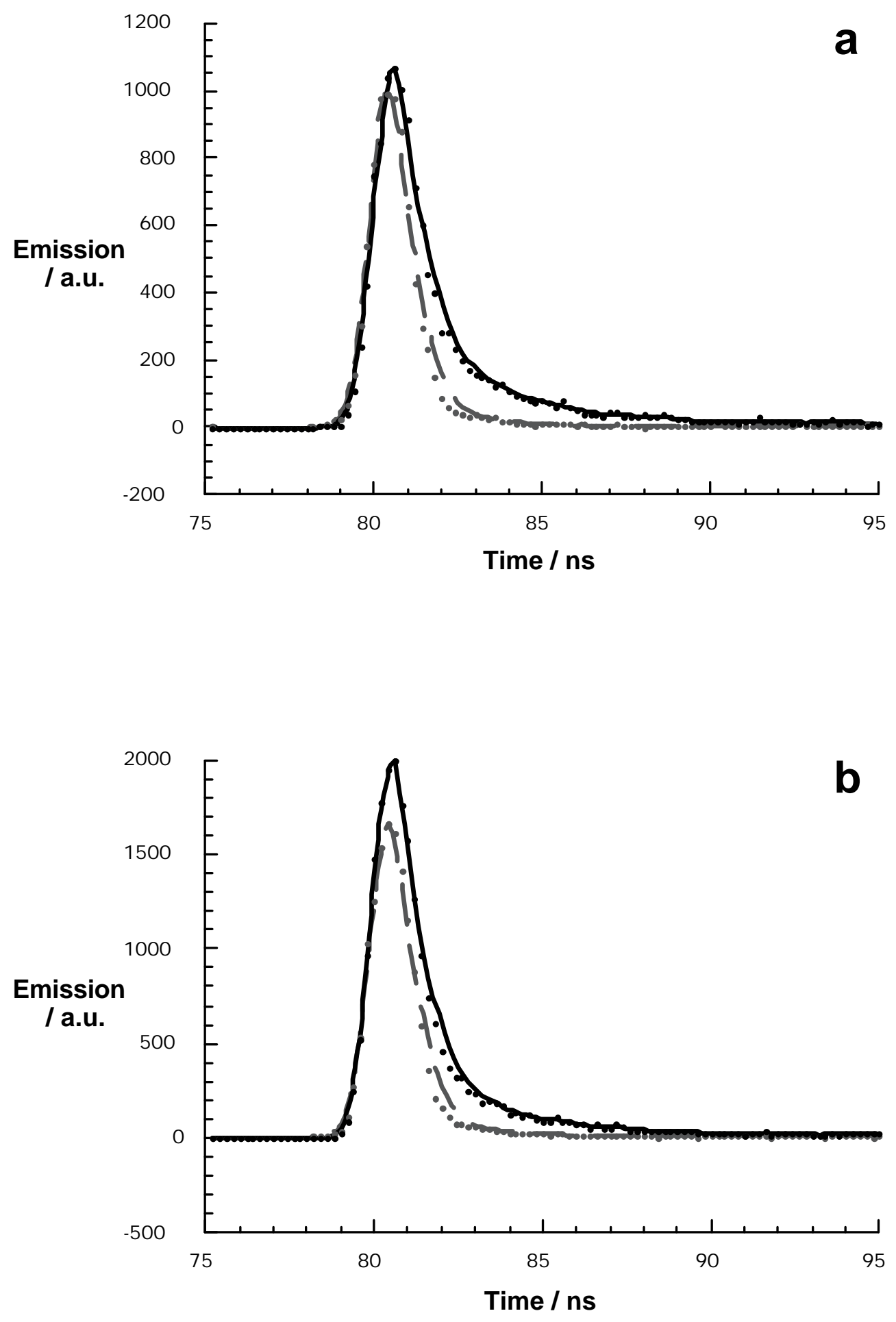

Figure S2: Fluorescence lifetime decays a) of $\mathbf{1}$ (monomer) and b) of $\mathbf{4}$ (pentamer) in THF (solid lines) - recorded at $720 \mathrm{~nm}$ - together with laser scatterer (dashed lines). 


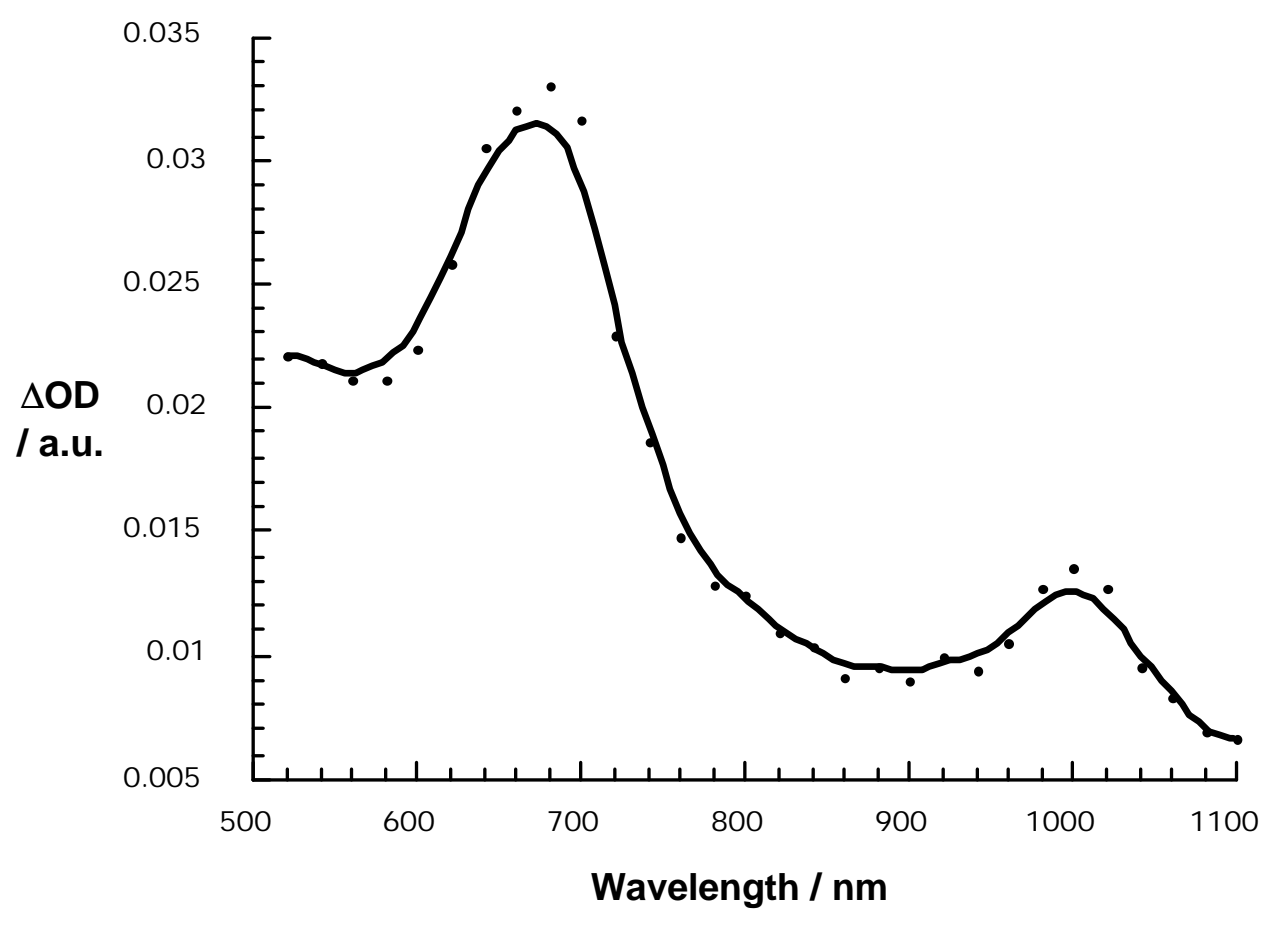

Figure S3: Differential absorption spectrum (visible and near-infrared) obtained upon nanosecond flash photolysis (355 nm) of $\sim 1.0 \times 10^{-5} \mathrm{M}$ solutions of $\mathbf{C}_{60}$-wire-exTTF (1) in nitrogen saturated THF with a time delay of $50 \mathrm{~ns}$ at room temperature. 


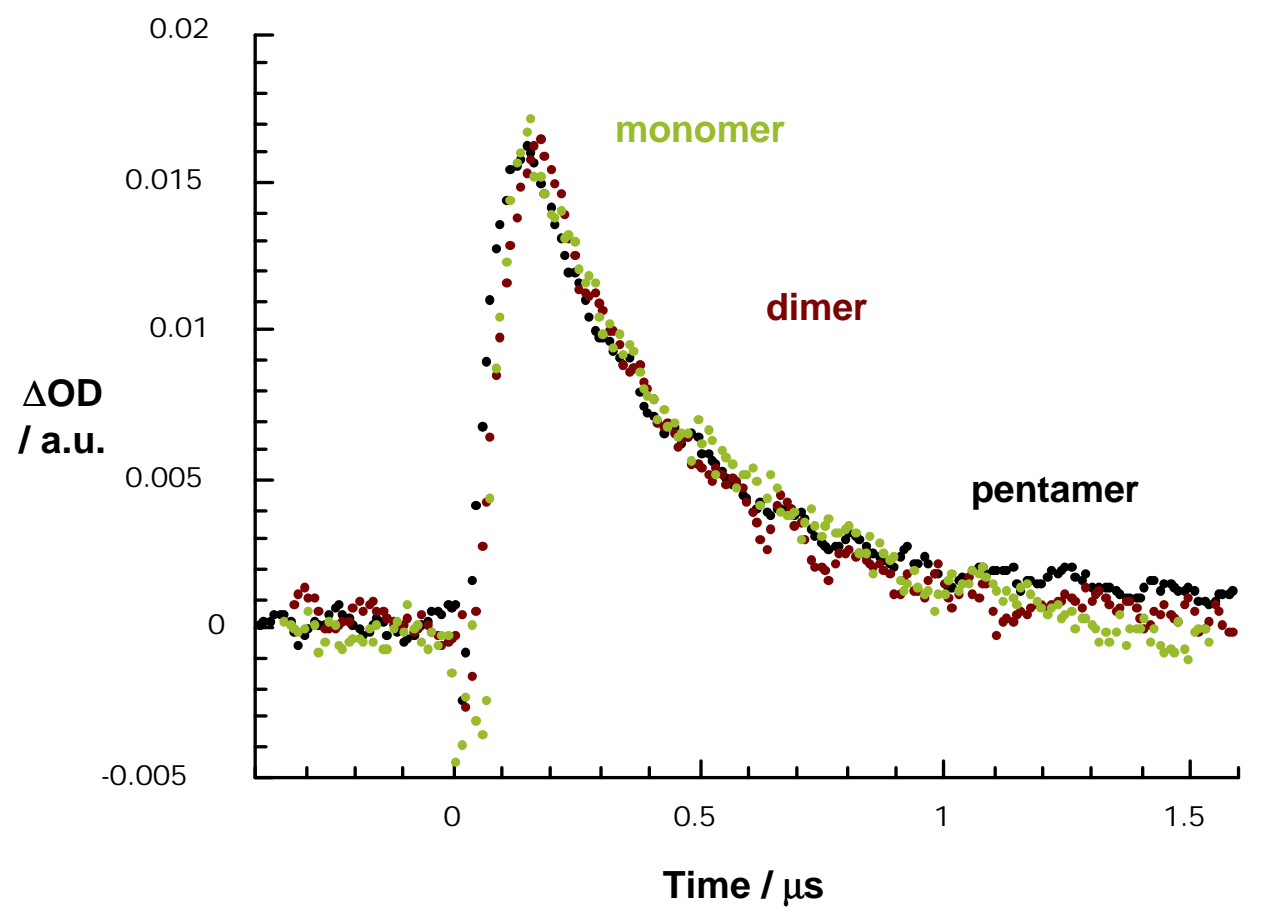

Figure S4a: Time-absorption profiles at $1000 \mathrm{~nm}$ monitoring the $\mathrm{C}_{60}{ }^{-}$decay dynamics in $\mathbf{1}$ (monomer), $\mathbf{2}$ (dimer) and $\mathbf{4}$ (pentamer) obtained upon nanosecond flash photolysis (355 nm) of $\sim 1.0 \times 10^{-5} \mathrm{M}$ deoxygenated benzonitrile solutions. 


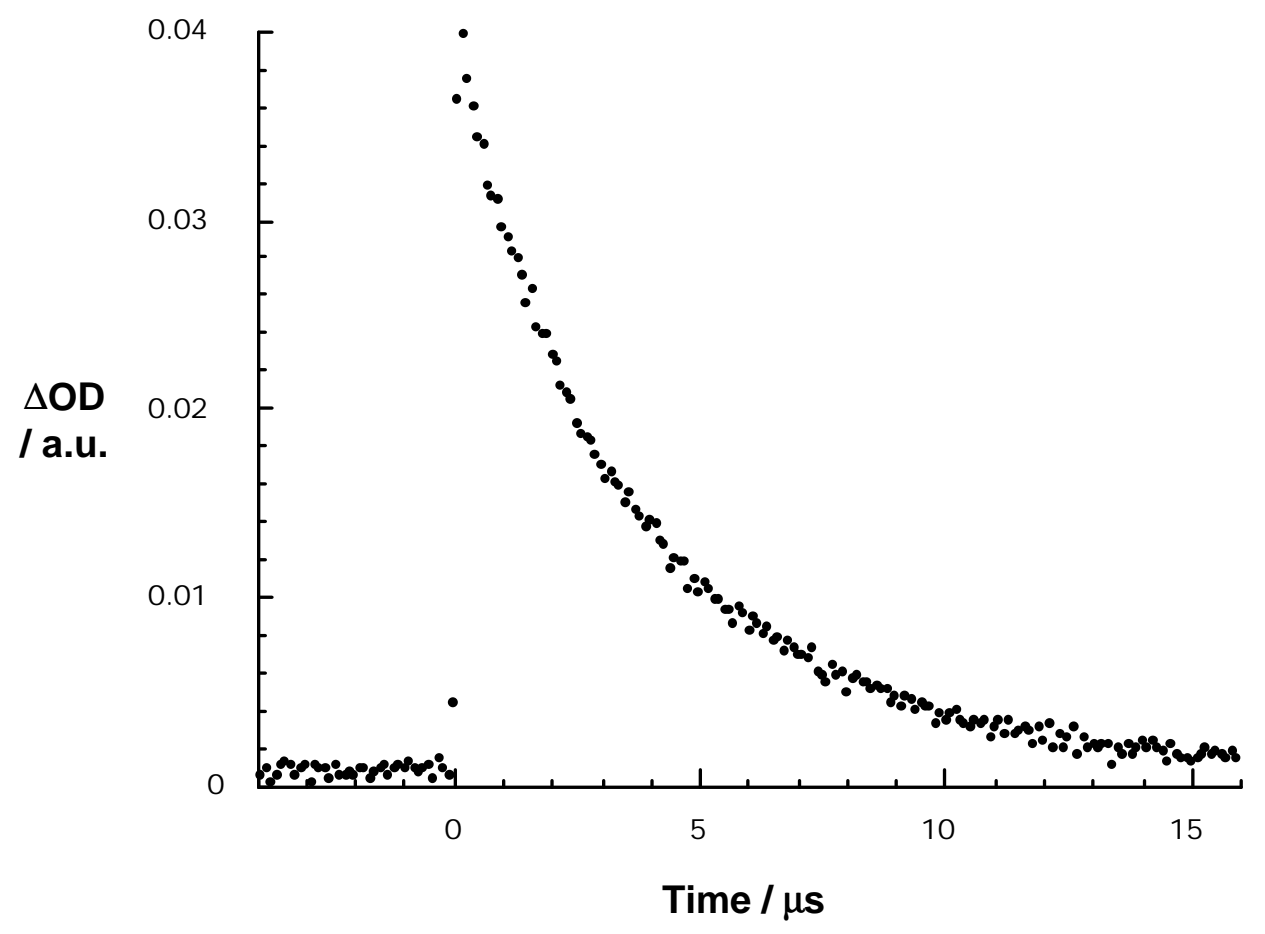

Figure S4b: Time-absorption profiles at $1000 \mathrm{~nm}$ monitoring the $\mathrm{C}_{60}{ }^{--}$decay dynamics in 5 (heptamer) obtained upon nanosecond flash photolysis $(355 \mathrm{~nm})$ of $\sim 1.0 \times 10^{-5} \mathrm{M}$ deoxygenated benzonitrile solutions. 
Table S1: Photophysical Features of $\mathrm{C}_{60}$-oligomer-exTTF Systems

\begin{tabular}{|c|c|c|c|c|c|c|c|c|c|c|}
\hline & \multicolumn{4}{|c|}{ THF } & \multicolumn{4}{|c|}{ benzonitrile } & \multicolumn{2}{|c|}{ DMF } \\
\hline & $\begin{array}{c}\text { fluore-scence }^{\text {lifetime }}{ }^{\text {a }} \\
\text { [ns] }\end{array}$ & $\begin{array}{c}\begin{array}{c}\text { singlet } \\
\text { lifetime }\end{array} \\
\text { bns] }\end{array}$ & $\begin{array}{c}\text { radical pair }^{\text {difetime }}{ }^{\mathbf{c}} \\
\text { [ns] }\end{array}$ & $\begin{array}{c}\text { electronic } \\
\text { coupling }\end{array}$ & $\begin{array}{c}\text { fluore- } \\
\text { scence } \\
\text { lifetime } \\
\text { [ns] }\end{array}$ & $\begin{array}{c}\text { singlet } \\
\text { lifetime }^{\mathbf{b}} \\
{[\mathrm{ns}]}\end{array}$ & $\begin{array}{c}\text { radical pair } \\
\text { lifetime }^{\mathbf{c}} \\
\text { [ns] }\end{array}$ & $\begin{array}{c}\begin{array}{c}\text { electronic } \\
\text { coupling }\end{array} \\
{\left[\mathrm{cm}^{-1}\right]}\end{array}$ & $\begin{array}{c}\text { fluore- } \\
\text { scence } \\
\text { lifetime }^{\mathbf{a}} \\
{[\mathrm{ns}]}\end{array}$ & $\begin{array}{c}\text { singlet } \\
\text { lifetime }^{b} \\
{[\mathrm{~ns}]}\end{array}$ \\
\hline 1 & 0.26 & 0.32 & 295 & 5.3 & 0.16 & 0.18 & 430 & 6.2 & $<<0.1$ & \\
\hline 2 & 0.27 & 0.28 & 321 & 6.1 & 0.16 & 0.2 & 465 & 6.1 & $<<0.1$ & 0.1 \\
\hline 3 & 0.27 & 0.33 & 365 & 6.9 & 0.17 & 0.2 & 499 & 5.9 & 0.09 & \\
\hline 4 & 0.3 & 0.35 & 411 & 5.8 & 0.18 & 0.21 & 557 & 5.5 & 0.11 & 0.13 \\
\hline 5 & 0.4 & 0.28 & 3190 & & 0.28 & 0.25 & 4350 & & 0.16 & 0.13 \\
\hline
\end{tabular}

a determined by monitoring the fullerene fluorescence decay at $720 \mathrm{~nm}$ in time-resolved fluorescence lifetime measurements

b determined by monitoring the fullerene singlet-singlet absorption at $900 \mathrm{~nm}$ in time-resolved transient absorption measurements

c determined by monitoring both features of the radical ion pair state, that is, the $660 \mathrm{~nm}$ absorption maximum of the one-electron oxidized donor (exTTF) and the $1000 \mathrm{~nm}$ absorption maximum of the one-electron reduced acceptor $\left(\mathrm{C}_{60}\right)$ in time-resolved transient absorption measurements

d determined from the following relation for a non-adiabatic electron transfer:

$k_{\mathrm{et}}=\left(\frac{4 \pi 3}{\mathrm{~h}^{2} \lambda \mathrm{k}_{\mathrm{B}} \mathrm{T}}\right)^{\frac{1}{2}} \mathrm{~V}^{2} \exp \left[\frac{-\Delta \mathrm{G} \#}{\mathrm{k}_{\mathrm{B}} T}\right]$

$h \quad=\quad$ Planck constant

$k_{\mathrm{b}} \quad=\quad$ Boltzmann constant $(0.025 \mathrm{eV}$ at $298 \mathrm{~K})$

$T \quad=\quad$ absolute temperature $(298 \mathrm{~K})$

$\lambda=$ reorganization energy $(1 \mathrm{eV})$

$G^{\#}=$ Gibbs activation energy, determined from the following relation:

$\Delta \mathrm{G}_{\mathrm{cs}}{ }^{\#}=\frac{\left(\Delta \mathrm{G}_{\mathrm{cs}}+\lambda\right)^{2}}{4 \lambda}$ 